\title{
The intricate interplay among body weight, stress, and the immune response to friend or foe
}

\author{
Lawrence Steinman, ${ }^{1}$ Paul Conlon, ${ }^{2}$ Rich Maki, ${ }^{2}$ and Alan Foster ${ }^{2}$ \\ ${ }^{1}$ Interdepartmental Program in Immunology and Department of Neurological Sciences, Stanford University, Stanford, California, USA \\ ${ }^{2}$ Neurocrine Biosciences, San Diego, California, USA
}

J. Clin. Invest. 111:183-185 (2003). doi:10.1172/JCI200317622.

We all know that when we suffer from fever we feel sleepy and do not feel like eating. Our mothers and grandmothers provided sound wisdom during our bouts of illness with fever. They recommended rest and tried to make us eat, advocating folk remedies like chicken soup or various teas. The physiologic roles of mediators of fever such as IL-1, TNF- $\alpha$, IL-6, and prostaglandins account for some of the physiological changes in the sleep/wake cycle and in appetite $(1,2)$. Prostaglandins are involved in the induction of sleep, while TNF- $\alpha$ triggers loss of appetite and pyrexia. However, we are now learning that the interconnections between molecules that modulate brain function and those that mediate the immune response are more intertwined than we had previously imagined.

\section{Neuroendocrine modulation of the immune response}

Leptin, a molecule that is critical in the regulation of energy balance and body weight, is a strong regulator of Th1 autoimmunity $(3,4)$. This is one of many examples of redundancy and overlapping roles of molecules within neuroendocrine systems and the immune system. For example, it has been shown

\footnotetext{
Address correspondence to: Lawrence Steinman, Interdepartmental Program in Immunology and the Department of Neurological Sciences, Stanford University, B002 Beckman Center for Molecular Medicine, Stanford, California 94205, USA. Phone: (650) 725-6401; Fax: (650) 725-0627; E-mail: steinman@stanford.edu.

Conflict of interest: The authors have declared that no conflict of interest exists. Nonstandard abbreviations used: corticotropin-releasing factor (CRF); $\alpha$-melanocyte-stimulating hormone $(\alpha-\mathrm{MSH})$; stearoyl CoA desaturase-1 (SCD-1).
}

that corticotropin-releasing factor (CRF), a master regulator of hypothalamic and pituitary function, has an autonomous effect on the immune system. CRF can downregulate Th1 autoimmunity $(2,5)$. The neuroendocrine system can have potent effects on the immune system. Basic behaviors such as fasting have potent influences on the induction of Th1 autoimmunity. A short fast can circumvent an attack of autoimmune paralysis (4). Thus, while "feeding a cold" may have salutary effects in combating a viral infection, starving an autoimmune disease may protect against immune damage.

While "simple" acts such as fasting influence immunity, complicated states like pregnancy modulate autoimmune diseases as strongly as any known drug $(2,6)$. Gender itself has an impact on autoimmunity, females being far more susceptible than males to diseases like systemic lupus erythematous, rheumatoid arthritis, autoimmune thyroiditis, and MS (2). Interestingly, leptin is produced to a much greater extent by females than by males (3). This may in part account for the increased susceptibility of females to many organ-specific autoimmune diseases. However, CRF activity in the hypothalamic pituitary axis is generally higher in females. Given the inhibitory effect of CRF on autoimmune disease (5), an apparent paradox emerges, probably masking another layer of complexity, unexplained for now.

\section{Leptin and CRF downregulate Th1 autoimmunity}

In the current issue of the $J C I$, Matarese and colleagues describe the potent effects of leptin on Th1 autoimmunity, including the differential production of this molecule in females and the remarkable beneficial effect of a short fast on downregulating an incipient attack of autoimmunity (4). The authors demonstrate that leptin is produced at the site of autoimmune pathology. Neurons producing leptin may actually alter immune function, indicating direct neural control of an immune response $(3,4)$. One possible explanation for the link between leptin and control of the immune system is that during starvation, which results in low levels of leptin, the organism must conserve energy, and reduced leptin levels force the organism to reduce reproductive and thermogenic activities (7) while suppressing the immune system (8). It is worth noting that starvation also increases CRF expression, which also downregulates autoimmunity (5). CRF in turn upregulates cortisol, which also acts to dampen autoimmunity, though the effects of CRF on autoimmunity are still apparent even after adrenalectomy (5). Thus starvation, at least in the short term, has beneficial effects on autoimmunity, probably via the roles of leptin and CRF. The question also arises as to whether obesity and autoimmunity are comorbid conditions, given the fact that leptin levels are highly elevated in obese subjects.

Leptin is a member of the helical cytokine family, which includes IL-6, IL-11, IL-12, leukemia inhibitory factor, GCSF, ciliary neurotrophic factor, and oncostatin $M(9,10)$. The leptin receptor is a member of the class I cytokine receptor family, which includes gp-130, the common signal transducing component for the IL-6related family of cytokines (9). Leptin mediates food intake, metabolism, and reproductive behavior. Leptin receptors 


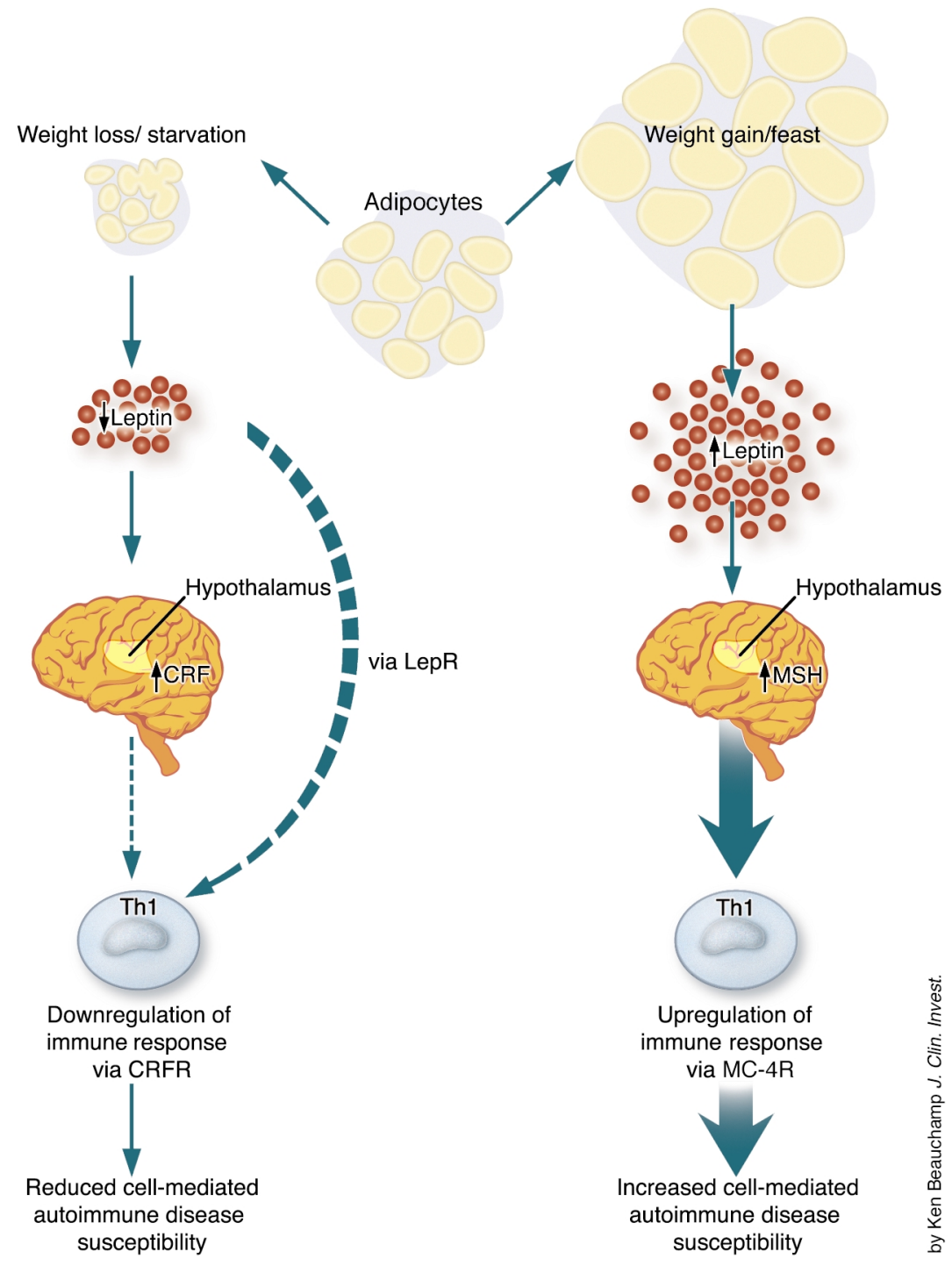

Figure 1

Body fat and the autoimmune response. Decreases in body fat or starvation increase CRF levels and decrease leptin levels. Increased CRF downregulates Th1 immunity (5) as does decreased leptin (4). Other molecules of the $\alpha$-MSH family stimulate Th1 T cells via their MC-4 receptor. CRFR, CRF receptor; LepR, leptin receptor.

are present on $\mathrm{CD} 4^{+} \mathrm{T}$ cells, where leptin mediates Th1 immunity. Mice with a deletion in the leptin gene $(o b / o b$ mice) have a high level of thymocyte apoptosis with a marked reduction in the size and cellularity of the thymus (11). T cells in these mice produce minimal IFN- $\gamma$ and a moderate amount of IL-4, resulting in an inability to generate a Th1 response while enhancing a Th2 phenotype $(3,4,8)$.

\section{Neuropeptides involved in body weight are found at sites of autoimmunity}

There are indications that other neuroendocrine mediators such as the melanocortins $(12,13)$ and CRF $(2$, level of the pro-opiomelanocortinexpressing neurons in the arcuate nucleus of the hypothalamus, which are activated by leptin to release $\alpha$-MSH (15). Apparently, this interplay of neuropeptides with regards to hypothalamic function may be reproduced at sites of autoimmune pathology (Figure 1). Largescale analysis of gene transcripts in MS lesions revealed that leptin, melanocortin 4 receptor, and ACTHreceptor are elevated at the site of inflammation in the brain $(12,13)$. All three of these neuropeptides leptin, melanocortin 4, and ACTH have pleiotropic roles in neuroendocrine and immune physiology.

The microsomal enzyme, stearoyl CoA desaturase-1 (SCD-1), is required for the biosynthesis of the monounsaturated fats, palmitoleate and oleate from saturated fatty acids (16). SCD-1 RNA levels are highly elevated in untreated $o b / o b$ mouse liver, and indeed SCD-1 probably plays a decisive role in leptin's metabolic effects. Interestingly, SCD-1 was downregulated in the MS brain as well (12), and both RNA levels and the activity of this enzyme are repressed by leptin (16). Ob/ob mice with mutations in SCD-1 are significantly less obese than $o b / o b$ controls (16). The role of leptin in autoimmune brain disease and in the immune system in general may be mediated by downregulation of this enzyme involved in the biosynthesis of monosaturated fats. Once again we witness the remarkable choreography of molecules related to body weight and energy metabolism and the parallel roles of these same molecules in the finely tuned immune response.

Earlier work showed that CRF, the key regulator of the stress response in the hypothalamic-pituitary-adrenal axis, or urocortin, a naturally occurring paralogue of CRF, acting directly on $\mathrm{T}$ cells in adrenalectomized mice, ameliorated experimental autoimmune encephalomyelitis. These effects were blocked by antagonists of CRF (5). Thus CRF, like leptin, is produced by the brain and may act directly on the immune system. The expression of CRF itself can be regulated by cytokines, adding yet another layer of complexity and a further target for intervention. Another neuropeptide, ACTH, a key 
mediator of the stress response produced in the pituitary gland, has been used for over 40 years to treat MS. ACTH receptor is expressed in the MS lesion itself (12).

These results imply that in autoimmunity, stress may be beneficial, and that short-term starvation may help reverse disease. In fighting microbial infections, perhaps stress is detrimental and eating is recommended, but in the case of autoimmunity, the opposite holds true: stress is actually good for you, and starvation is helpful. Grandmother's advice may have to be refined depending on whether the immune system is attacking friend or foe.

\section{Acknowledgments}

The authors thank Wylie Vale for his insights and input.
1. Steinman, L. 1993. Connections between the immune system and the nervous system. Proc Natl. Acad. Sci. USA. 90:7912-7914.

2. Steinman, L. 1993. Autoimmune disease. Sci. Am 269:106-114.

3. Matarese, G., et al. 2001. Requirement for leptin in induction and progression of experimenta autoimmune encephalomyelitis. J. Immunol. 166:5909-5916.

4. Sanna, V., et al. 2003. Leptin surge precedes onset of autoimmune encephalomyelitis and correlates with development of pathogenic T cell responses. J. Clin. Invest. 111:241-250. doi:10.1172/JCI200316721.

5. Poliak, S., et al. 1997. Stress and autoimmunity: the neuropeptides corticotropin releasing factor and urocortin suppress encephalomyelitis via effects on both the hypothalamic-pituitary-adrenal axis and the immune system. J. Immunol. 158:5751-5756.

6. Langer-Gould, A., Garren, H., Slansky, A., Ruiz, P.J., and Steinman, L. 2002. Late pregnancy suppresses relapses in experimental autoimmune encephalomyelitis: evidence for a suppressive pregnancy-related serum factor. J. Immunol. 169:1084-1091.

7. Ahima, R.S., et al. 1996. Role of leptin in the neuroendocrine response to fasting. Nature 382:250-252.

8. Lord, G.M., et al. 1998. Leptin modulates the T-cell immune response and reverses starvation-induced immunosuppression. Nature. 394:897-901.

9. Baumann, H., et al. 1996. The full-length leptin receptor has signaling capabilities of interleukin 6-type cytokine receptors. Proc. Natl. Acad. Sci. USA. 93:8374-8378.

10. Zhang, F., et al. 1997. Crystal structure of the obese protein leptin-E100. Nature. 387:206-209.

11. Howard, J., et al. 1999. Leptin protects mice from starvation-induced lymphoid atrophy and increases thymic cellularity in $o b / o b$ mice. J. Clin. Invest. 104:1051-1059.

12. Lock, C., et al. 2002. Gene microarray analysis of multiple sclerosis lesions yields new targets validated in autoimmune encephalomyelitis. Nat Med. 8:500-508.

13. Steinman, L., Martin, R., Bernard, C.C.A., Conlon, P. and Oksenberg, J.R. 2002. Multiple sclerosis: deeper understanding of its pathogenesis reveals new targets for therapy. Ann. Rev. Neurosci. 25:491-505.

14. Marks, D.L., Ling, N., and Cone, R.D. 2001. Role of the central melanocortin system in cachexia. Cancer Res. 61:1432-1438.

15. Cowley, M.A., et al. 2001. Leptin activates anorexigenic POMC neurons through a neural network in the arcuate nucleus. Nature. 411:480-484.

16. Cohen, P., et al. 2002. Role for stearoyl-CoA desaturase-1 in leptin-mediated weight loss. Science. 297:240-243. 